\title{
Effect of unsharpness on the result of thermovision diagnostics of electronic components
}

\author{
by K. DZIARSKI* and A. HULEWICZ**
}

\author{
*Poznań Univ. of Technology, 60-965, Piotrowo 3A Str., Poznań, Poland, Krzysztof.Dziarski@put.poznan.pl \\ ** Poznań Univ. of Technology, 60-965, Piotrowo 3A Str., Poznań, Poland, Arkadiusz.Hulewicz@put.poznan.pl
}

\begin{abstract}
This work presents the effect of thermogram unsharpness on the result of thermovision temperature measurement. Thermograms have been made using the thermal imaging camera operating in Long-Wave Infrared bandwidth, fitted with macro lens. The element, temperature of which was measured was the $2.3 \mathrm{~mm} \times 2.1 \mathrm{~mm}$ temperature sensor placed in a housing for through-hole installation. The sharpness of thermograms was determined using the selected measures. The determined sharpness of thermograms was verified through comparing with responses of one hundred and seven respondents. The relationship was described between the thermogram sharpness and the value of absolute thermovision temperature measurement error.
\end{abstract}

\section{Introduction}

Managing the temperature of electronic components is a difficult task. Too high value of a temperature of semiconductor junction can cause its malfunction and even damage. Too low temperature of an electronic element may indicate that the element can be more heavily loaded and that the cooling system of an electronic element has been oversized. Thus, the selection of a smaller and cheaper radiator, for example, is possible. The literature describes many ways allowing to determine the temperature of electronic components. Methods are known, consisting in applying a thermocouple to an element housing. It is worth noting that in the case of this method, the result of temperature measurement depends, among others, on the thermojunction - housing thermal combination and on the force of applying the thermojunction to the housing of an electronic element. Therefore, another method is currently used to determine the temperature of a semiconductor junction on the basis of TSP (Thermal Sensitive Parameter). The TSP is a parameter the value of which changes along with the temperature of semiconductor element. It is worth noting that applying the method that allows determining the temperature of a semiconductor element on the basis of a known TSP value is not entirely free of problems. When applying this method, it is necessary to know the relationship between the temperature of a semiconductor element and the value of the selected TSP. This requires performing long-term laboratory tests. In addition, the interference in the circuit housing of the test element is required. The above problems can be avoided by using thermovision. This non-contact method allows measuring the temperature of a housing containing a semiconductor junction on the basis of IR radiation emitted by this housing. The advantage of using the thermovision is the possibility of obtaining a distribution of temperatures on the housing surface. This allows detecting the so-called hotspots, which are usually small areas with increased temperatures. Hot spots can be characterized by dimensions of several dozen micrometres. Many modern electronic components have small housings, in the order of millimetres (e.g. SOT 232). It is necessary to use a macro lens to observe such elements. Thermovision measurements of small objects can be troublesome as it is difficult to obtain a sharp thermogram. It is necessary to carefully select the distance $d$ between the lens and the observed surface and the angular position $\alpha$ of the focusing adjustment ring. Changing the distance $d$ even by $1 \mathrm{~mm}$ can cause a large increase in thermogram unsharpness. It is related to a small depth of field. When observing surfaces staying at different distance from the lens, there is a variation in the sharpness of these surfaces.

The paper presents the effect of lack of sharpness of the registered thermogram on the result of thermovision measurement of the surface temperature of an electronic element (item 2). During the tests, an electronic element was being observed with a thermal imaging camera with an additional macro lens. The measuring system was presented and the method of stabilizing the conditions prevailing during the making of thermograms was discussed (item 3) Sharpness measures, which were used to determine the sharpness of the recorded thermograms were described (item 4) The results of thermogram sharpness measurements (item 5) were presented and the method to select one of them was discussed (item 6). The results of studies on the effect of thermogram unsharpness on the result of temperature measurement with a thermal imaging camera were presented (item 7).

\section{Sharpness of the thermogram recorded}

The recorded thermogram is perceived by the observer as a sharp thermogram, when the edges of areas assigned to fragments of the observed surface at different temperatures are not blurred [1]. Along with the increase in blurring of edges of these areas, the observer begins to perceive the observed thermogram as out of focus. The sharpness of thermogram can be adjusted by changing the distance $d$ [2] and changing the $\alpha$ [3]. As a result, the change 
in these two values affects the blurring of edges on the observed thermogram, and thus the thermogram sharpness assessment. Sharpness is a subjective impression - it depends on the observer's individual characteristics. For this reason, the selection of distance $d$ and angular position $\alpha$ will also depend on the observer's visual impressions.

The thermogram is perceived as sharp (the edges are not blurred) when the IR rays reaching the camera lens refract in the objective lens in such a way that they intersect in the matrix of the IR detectors [4]. In this case, the image emerges on the detector matrix. Only then the correct amount of IR radiation is absorbed by detectors. Consequently, the error component related to the thermogram unsharpness is the smallest. Otherwise, when the IR rays do not intersect on the detector matrix, the image emerges ahead or behind the detector matrix. Detectors absorb the incorrect amount of energy. Consequently, the temperature value indicated by the camera may differ from the actual value.

The point of intersection of the IR rays entering the lens depends on the value of angle of refraction of radiation $\beta$ in the camera lens. On the other hand, the value $\beta$ depends on the position of lens relative to the matrix of the IR detectors and the surface observed. Therefore, a change in $d$ will result in a change in $\beta$. In the case of thermal imaging cameras, the position of lens changes along with the change of $\alpha$. The change in $\beta$ can result in a change in the amount of energy supplied to the detector matrix. Consequently, the temperature value read from the unsharp thermogram can significantly differ from the correct temperature value. Also, it may not be possible to detect a hot spot on the basis of unsharp thermogram. [5]

Along with the change in thermogram sharpness, the indication of thermal imaging camera changes. The relationship between the result of thermovision temperature measurement and the sharpness of thermogram was noticed during the observation of electronic components placed in the SMD housings and small housings intended for THT (Through-Hole Technology) installation. Due to the specifics of the work conducted, it was decided to observe an electronic element with the shape and dimensions similar to the SMD housings. At the same time, it was assumed that such an element must have a surface which is easy to observe by thermovision and allows for electrical temperature measurement. These requirements are met by a Pt1000 resistance temperature sensor with dimensions of $2.3 \times 2.1 \mathrm{~mm}$ [6].

In order to illustrate the relationship between the result of thermovision temperature measurement $\vartheta_{m}$ and the sharpness of thermogram, three thermograms of a Pt 1000 sensor for different lens - sensor distances $d$ were shown in Fig. 1. The thermograms were recorded with a Flir E50 thermal imaging camera [7] with an additional macro Close-up Lens 2x 197214 [8] under the same conditions. In order to adjust the effect of factors that may affect the result of temperature measurement, the respective values were entered into the memory of thermal imaging camera $(\varepsilon=0.64$, reflected temperature $26.9^{\circ} \mathrm{C}$, air temperature $26.9^{\circ} \mathrm{C}$, relative humidity $35 \%$, exposure time longer than $3 \mathrm{~s}$.). Despite of including the conditions prevailing during the making of thermograms and the constant temperature $\vartheta$,of the observed electronic component, different indications of the thermal imaging camera were observed on thermograms with different sharpness. The $\vartheta_{m}$ value is the result of temperature measurement at SP1 point.

a) $\vartheta_{m}=100.8^{\circ} \mathrm{C}$ for $d=W D=33 \mathrm{~mm}$

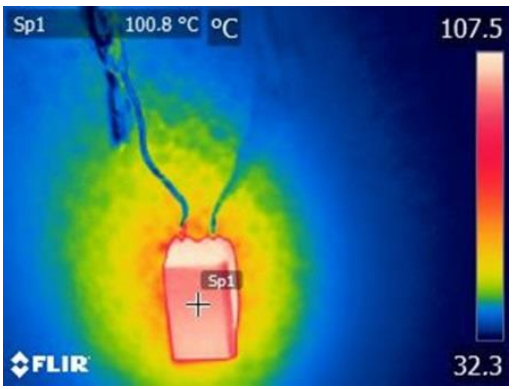

b) $\vartheta_{m}=100.5^{\circ} \mathrm{C}$ for $d=32 \mathrm{~mm}$

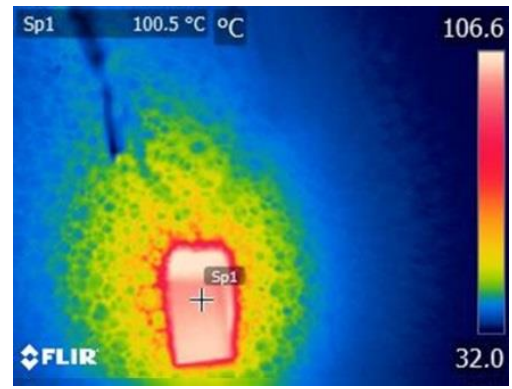

c) $\vartheta_{\mathrm{m}}=97.4^{\circ} \mathrm{C}$ for $d=25 \mathrm{~mm}$

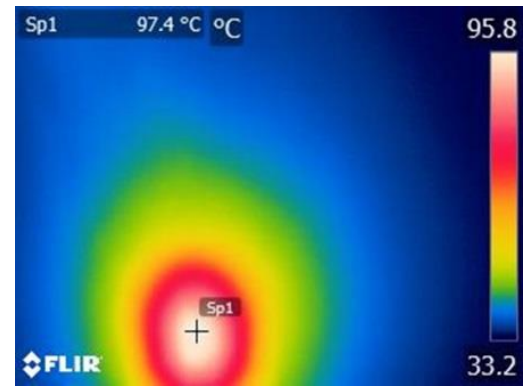

Fig. 1. Thermograms of the Pt 1000 sensor for different distances d between the lens and the sensor; WD - Work Distance

\section{Laboratory test bench for testing the effect of unsharpness on the result of temperature measurement}

In order to study the phenomenon shown in Fig. figure 1, it was decided to determine the relationship between the unsharpness of the recorded thermogram of an electronic element and the result of thermovision measurement of the surface temperature of the element. It was decided to check to what extent can the thermogram made with the use of an additional macro lens be unsharp, so that the result of the thermovision measurement of temperature of the surface of an electronic component was reliable. The analysis of this phenomenon can be reduced to determining the relationship between the sharpness of thermogram determined on the basis of the selected measure and the values of distance $d$ and angular position $\alpha$. In order to determine such relationship, the appropriate thermograms were needed to be obtained. The laboratory test bench was made for this reason with an E50 thermal imaging camera with additional macro Close-up Lens $2 \times 197214$ [3]. The camera was placed on a tripod allowing to change the distance $d$ with an $\times$ step equal to $1 \mathrm{~mm}$. The camera was placed inside the chamber made of plexiglass with dimensions of $45 \mathrm{~cm} \times 45 \mathrm{~cm} \times 33 \mathrm{~cm}$. For the automatic setting of angular position $\alpha$ of the focusing adjustment ring, a stepper motor working with the PLC with the 
HMI panel was used. The drive from the stepper motor shaft was transmitted to the ring by means of a rubber strap. To monitor the measurement conditions inside the chamber a humidity and temperature sensor was placed. For optical isolation of the test bench, the walls of chamber and the stepper motor housing were lined with porous black polyurethane foam. The Flir Tools program was used for the archiving of thermograms The making of thermograms allowed communicating the thermal imaging camera with the PC with the Flir Tools software via a USB interface. The design of the laboratory test bench is shown in figure 2 .

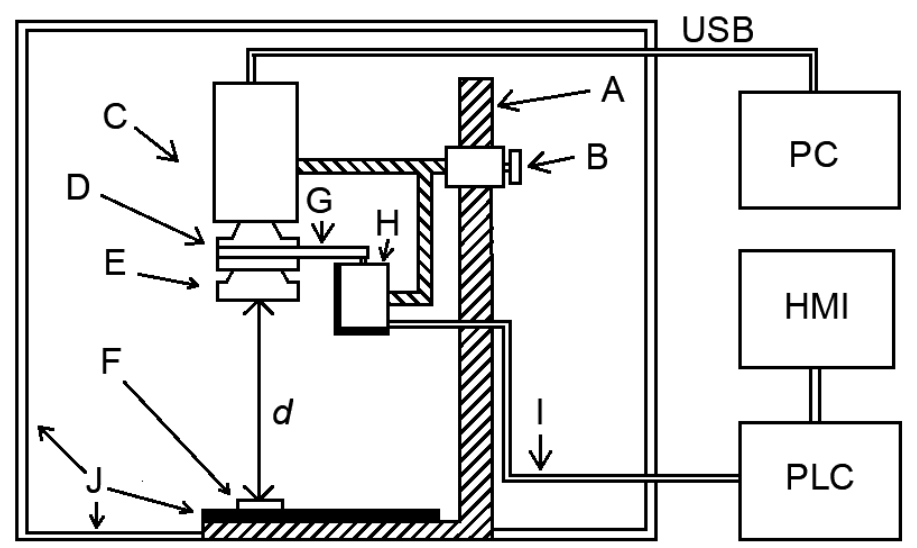

Fig. 2. Design of the laboratory test bench: $A$ - Tripod B - Micrometre screw for setting the distance $d, C$ -

Thermal imaging camera, D - Basic thermal imaging camera lens, $E$ - Additional macro lens, F - Observed Pt1000 sensor, G - Rubber strap, $H$ - Stepper motor I - Wire between the engine and the measurement system, $\mathrm{J}$ - Polyurethane foam

\section{Thermogram sharpness measures}

In order to determine the relationship between the sharpness of the recorded thermogram and the result of the thermovision temperature measurement, it was necessary to measure the sharpness of thermogram using an appropriate measure. The literature describes many mathematical measures of image sharpness (photographs). There are reports on the use of some of them to determine the sharpness of thermograms $[9,10]$. Measures that allow determining the image sharpness can be broken down into measures that determine the sharpness due to the result of the transformation and measures that use image variation in the spatial domain. As an example of a measure using the transformation, one can indicate the analysis of an image based on identification of distortions and verification of reliability of an image called DIIVINE (Distortion Identification-based Image INtegrity and Verity Evaluation) [11]. Another example of a transformation-based measure of sharpness is a measure based on LPC (Local Phase Coherence) [12]. An example of a measure using the variation of image content is a measure based on JNB (Just Noticeable Blur) edge width [13]. Other examples include a measure based on the probability of detecting the blur called CPBD (Cumulative Probability of Blur Detection) [14] and statistical methods for determining the sharpness of an image. Other examples that should be noted include the S3 (Spectral and Spatial Sharpness) measure [15], and the measure using the natural statistics of local luminance coefficients [16], magnitude of gradient [17]. During the research conducted, it was noticed that along with the change in the sharpness of the thermogram recorded, the boundaries between these areas of thermogram that represent surfaces of different temperatures are increasingly blurred. For this reason, it was decided to use the sharpness measures based on the variability of image content. An additional advantage of these measures is their easy implementation.

The simplest measure that allows determining the image sharpness on the basis of the variability of its content is variance. The image blurred has less variance than the sharp image [18]

$$
D^{2}=\frac{1}{M \cdot N} \sum_{x=0}^{M-1} \sum_{y=0}^{N-1}(f(x, y)-\mu)^{2}
$$

where:

$$
\mu=\frac{1}{M \cdot N} \sum_{x=0}^{M-1} \sum_{y=0}^{N-1} f(x, y)
$$


The measure using the vertical and horizontal image gradient is the Energy of Gradient (EOG). The more blurred the boundary between the two fragments of thermogram representing areas with different temperatures, the smaller the gradient. In turn, the higher the gradient value, the sharper the image. The EOG can be described by the equation Eq. (3) $[10,18,19]$ :

$$
\mathrm{EOG}=\sum_{x=1}^{M} \sum_{y=1}^{N}\left(f_{x}+f_{y}\right)
$$

The modified version of the energy of gradient of an image Energy of Laplacian (EOL) is the SF (Spatial Frequency). The results obtained using the SF presented in this paper were compared with values obtained using other measures. The $S F$ is the number of changes in the value of brightness on a unit segment of any fragment of an image. The $S F$ has been defined as [18]:

$$
S F=\sqrt{(R F)^{2}+(C F)^{2}}
$$

where $R F$ (Row Frequency) - frequency of the order, CF (Column Frequency) - column frequency

$$
\begin{aligned}
& R F=\sqrt{\frac{1}{M \cdot N} \sum_{x=1}^{M} \sum_{y=2}^{N}[f(x, y)-f(x, y-1)]^{2}} \\
& C F=\sqrt{\frac{1}{M \cdot N} \sum_{x=2}^{M} \sum_{y=1}^{N}[f(x, y)=f(x-1, y)]^{2}}
\end{aligned}
$$

Another of the measures used, which is based on the sharpness of edge of the recorded thermogram is the Energy of Laplacian (EOL). This measure is using the second function derivatives in both directions. The higher the value of the second derivative, the less blurred the boundary between areas representing a fragment with two different temperatures $[10,18]$.

$$
\mathrm{EOL}=\sum_{x=2}^{M-1} \sum_{y=2}^{N-1}\left(f_{x x}^{2}+f_{y y}^{2}\right)^{2}
$$

Nayar and Nakagawa noticed that the second derivatives in both directions may have opposite signs and cancel each other reciprocally. For this reason, in order to determine the sharpness, they proposed a new measure - the Sum of Modified Laplasian. The modified laplasian $M N$ has been described as $[10,18]$ :

$$
\nabla_{M L} f(x, y)=|2 f(x, y)-f(x-h, y)-f(x+h, y)|+|2 f(x, y)-f(x, y-h)-f(x, y+h)|
$$

The space (step) between the matrix elements representing the colour intensity of the thermogram is designated as $h$. In the works performed, step " $h$ " was always 1 . The SML can be determined by the equation:

$$
\mathrm{SML}=\sum_{i=x-N}^{x+N} \sum_{j=y-N}^{y+N} \nabla_{M L}^{2} f(i, j)^{2}, \nabla_{M L}^{2} f(i, j) \geq T
$$

Another measure based on the distribution of values around the edge is the Tenenbaum algorithm (Tenengrad). This method is based on determining the gradient magnitude using the Sobel operator $\nabla S(\mathrm{x}, \mathrm{y})$. The Sobel operator is one of the operators used for edge detection. The Tenengrad can be determined by the following equation $[10,18]$ : 


$$
\text { Tenengrad }=\sum_{x=2}^{M-1} \sum_{y=2}^{N-1}(\nabla S(x, y))^{2}, \nabla S(x, y)>T
$$

in which $T$ is the threshold of discrimination. The Sobel gradient magnitude can be expressed by:

$$
\nabla S(x, y)=\sqrt{\nabla S_{x}(x, y)^{2}+\nabla S_{y}(x, y)^{2}}
$$

while $\nabla S_{x}(x, y)$ and $\nabla S_{y}(x, y)$ can be expressed using equations:

$$
\begin{aligned}
& \nabla S_{x}(x, y)=\{-[f(x-1, y-1)+2 f(x-1, y)+f(x-1, y+1)]+[f(x+1, y-1)+2 f(x+1, y)]+ \\
& +[f(x+1, y+1)]\} \\
& \nabla S_{y}(x, y)=\{+[f(x-1, y-1)+2 f(x, y-1)+f(x+1, y-1)]+[f(x-1, y+1)+2 f(x, y+1)]+ \\
& +[f(x+1, y+1)]\}
\end{aligned}
$$

\section{Thermogram sharpness assessment}

Using the measures described in (1) - (12), the sharpness of thermograms gathered from all series was assessed. For the purpose of comparing the values of individual measures, they were normalized.

$$
V^{\prime}=\frac{V-V_{\min }}{V_{\max }-V_{\min }}
$$

where: $V^{\prime}-$ normalized value of sharpness measure, $V_{\min }$ - minimum value of sharpness measure in a given series, $V_{\max }$ maximum value of sharpness measure in a given series.

The normalized values of individual sharpness measures of thermograms from the selected series are shown in Fig. figure 3 (for series 3) and Fig. figure 4 (for series 5). In order to provide better readability, the values of measures determined on the basis of EOL, EOG and SF as well as on the basis of SML, Tenengrad and Variance have been set separately.

a) measures: EOL, EOG and SF

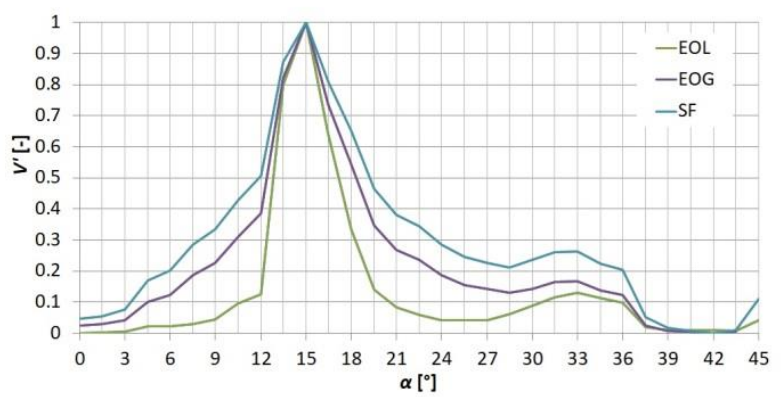

b) measures: SML, Tenengrad and Variance

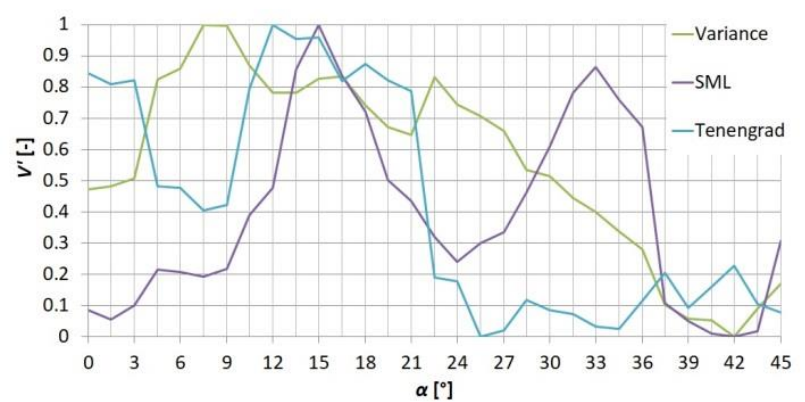

Fig. 3. Relationships $V^{\prime}=f(\alpha)$ for the sharpness measures used 
a) measures: EOG, EOL and SF

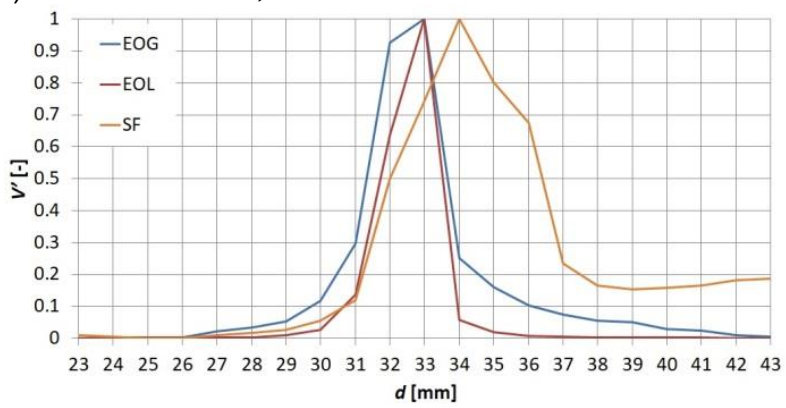

b) measures: SML, Tenengrad and Variance

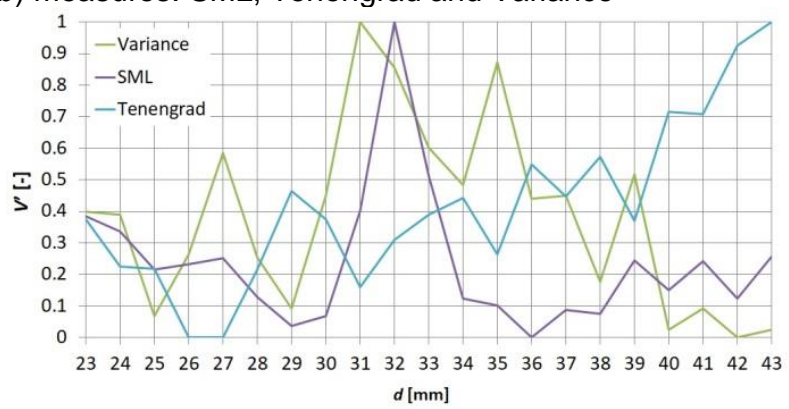

Fig. 4. Relationships $V^{\prime}=f(d)$ for the sharpness measures used

\section{Selection of thermogram sharpness measures}

The determined values of mathematical measures of thermogram sharpness (figure 3 and 4 ) are divergent. Therefore, it is necessary to select the best measure. It was assumed that the best measure would be the one that matches best the visual impressions of observers. Therefore, a survey was conducted with the participation of volunteer observers. They were shown the gathered thermograms and asked to indicate the most sharp thermogram in each of the six series. The 107 observers with different characteristics participated in the survey. The survey results obtained are shown in figure 5 .

a) $n=f(\alpha)$

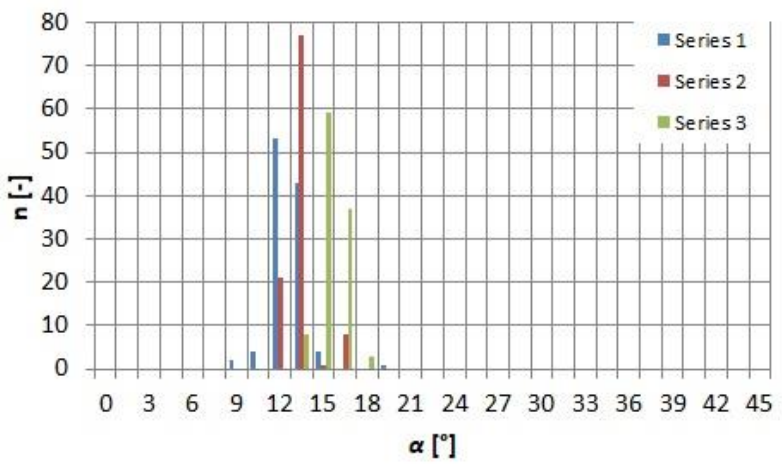

b) $n=f(d)$

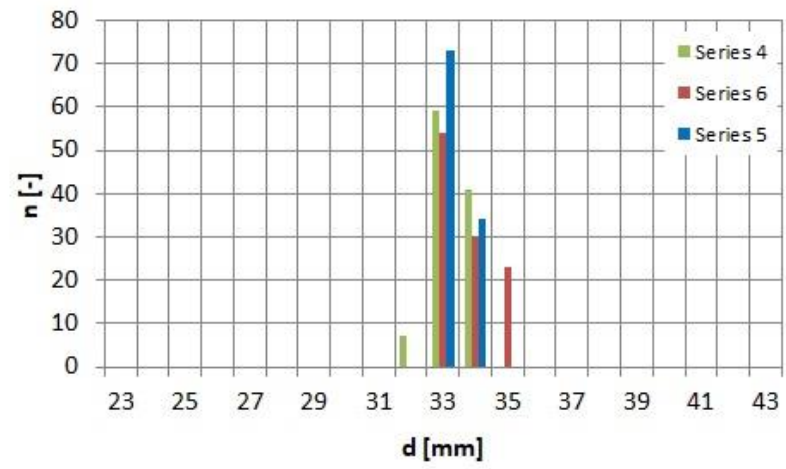

Fig. 5. Survey results of thermogram sharpness assessment by observers; $n$ - number of thermograms indicated as sharp

When comparing the results shown in figure 3 and 4 with relationships shown in figure 5 , it can be noticed that the greatest convergence occurs between indications of observers and results of sharpness obtained by means of measures using the derivatives in vertical and horizontal direction (EOG, EOL) and spatial frequency (SF). Other measures used (Tenengrad, SML and variance) show smaller convergence with observers' indications. Considering the results of surveys and the simplicity of implementation, the EOL measure was selected. In order to confirm the validity of selection of sharpness measure, it was decided to summarize in figure 6 the values of EOL measure and survey results for all series of measurements. 
b) $E O L=\mathrm{f}(\alpha), n=\mathrm{f}(\alpha)$
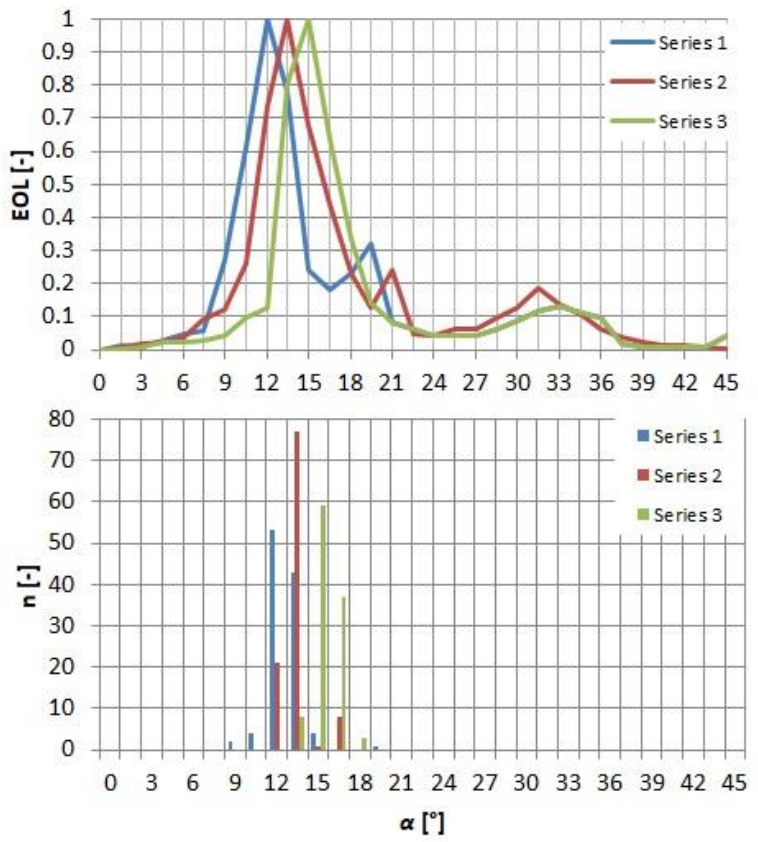

b) $E O L=\mathrm{f}(d), n=\mathrm{f}(d)$
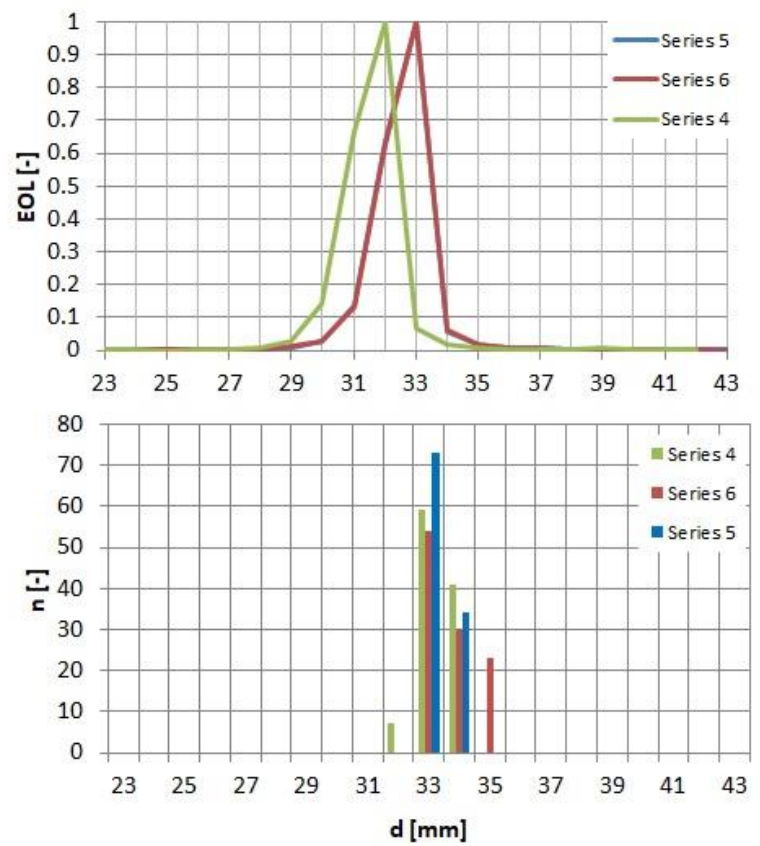

Fig. 6. Summary of relationship $E O L=f(\alpha)$ and $E O L=f(d)$ and survey results. In the case of relationship $E O L=f(d)$, the curves obtained for series 5 and 6 overlapped.

\section{Relationship between the result of thermovision temperature measurement and thermogram sharpness}

After the measurements at the laboratory test bench and selecting a mathematical sharpness measure, the effect of thermograms sharpness on the result of thermovision temperature measurement was determined. To present this effect, the relationship between the temperature measurement error $\Delta \vartheta_{m}$ and the distance $d$ and the angular position $\alpha$ was applied, where: [20]

$$
\Delta \vartheta_{m}=\vartheta_{m}-\vartheta_{s}
$$

The determined relationships $\Delta \vartheta_{m}=\mathrm{f}(\alpha)$ and $\Delta \vartheta_{m}=\mathrm{f}(d)$ were shown in figure 7 .

a) $\Delta \vartheta_{m}=\mathrm{f}\left(\alpha, \vartheta_{s}=101.6^{\circ} \mathrm{C}\right)$

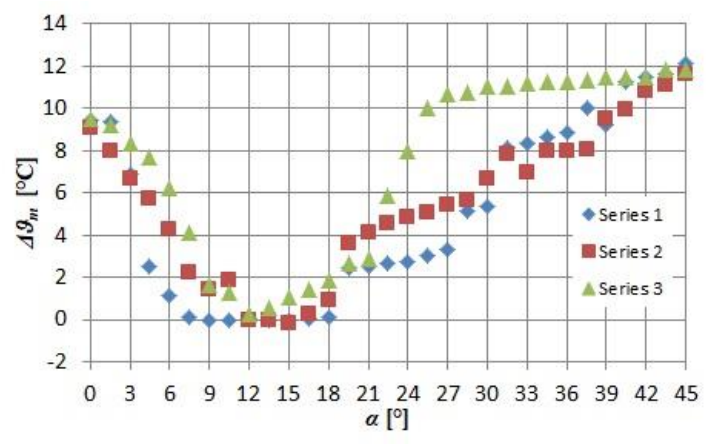

b) $\Delta \vartheta_{m}=\mathrm{f}\left(d, \vartheta_{s}=101.6^{\circ} \mathrm{C}\right)$

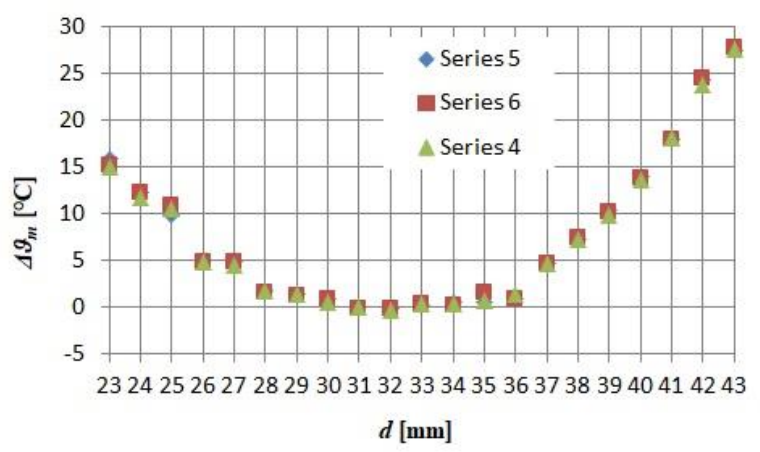

Fig. 7. Relationships $\Delta \vartheta_{m}=f(\alpha)$ and $\Delta \vartheta_{m}=f(d)$ determined for six series of measurements for $\vartheta_{s}=101.6{ }^{\circ} \mathrm{C}$. $W D=33 \mathrm{~mm}$. 
Obtainig the value of $\Delta \vartheta_{m}$ allowed determining the relationship between the normalized value of sharpness measure (EOL) and $\Delta \vartheta_{m}$. On the basis of this relationship, it is possible to answer the question to what extent (in the case of automatic sharpness assessment) the thermogram may be out of focus, so that the error of thermovision temperature measurement with the use of a macro lens was acceptably small. The relationships determined are shown in figure 8.

a) $\Delta \vartheta_{m}=\mathrm{f}\left(V^{\prime}, \vartheta_{s}=101.6^{\circ} \mathrm{C}, \alpha=\right.$ var, $d=$ const $)$

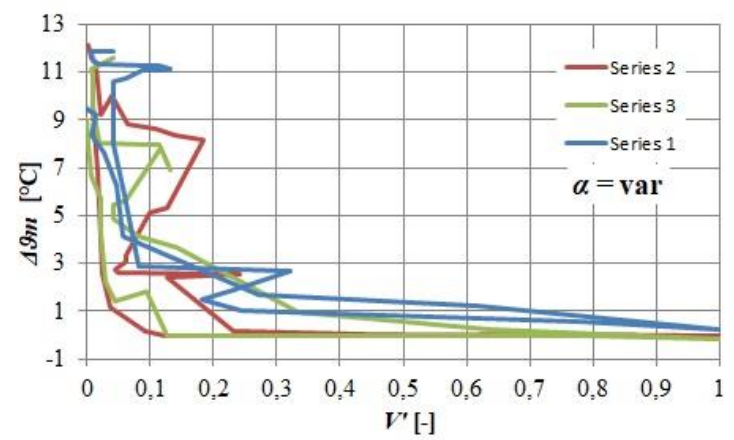

b) $\Delta \vartheta_{m}=f\left(V^{\prime}, \vartheta_{s}=101.6^{\circ} \mathrm{C}, \alpha=\right.$ const, $d=$ var $)$

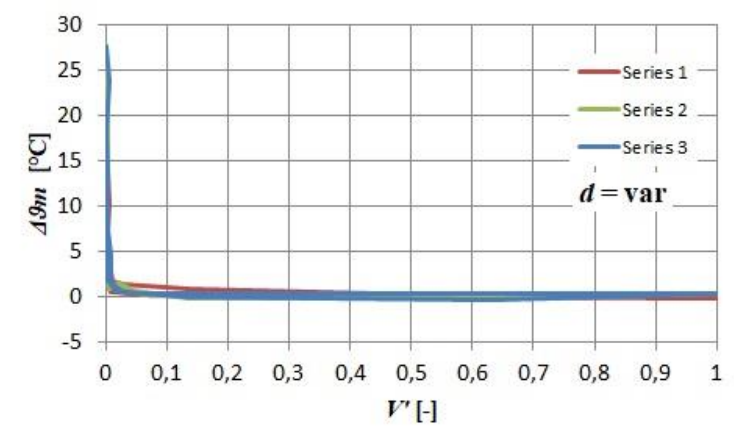

Fig. 8. Relationship $\Delta \vartheta_{m}=f\left(V^{\prime}\right)$ for variable values of distance $d$ and angular position $\alpha$; the normalized value of sharpness measure was determined for $E O L$

In order to facilitate the reference of relationships shown in figure 8 to actual thermograms, figure 9 shows the thermograms with determined sharpness. The sharpness of thermograms shown in figure 9 . was determined using the EOL. The EOL values were normalized in accordance with (13). The $\vartheta_{m}$ value was measured in the middle of the element.

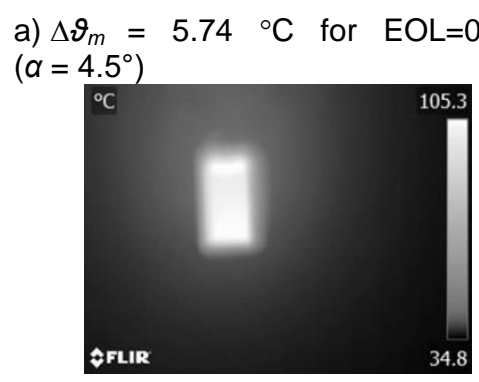

d) $\Delta \vartheta_{m}=4.76^{\circ} \mathrm{C}$ for $\mathrm{EOL}=0.032$

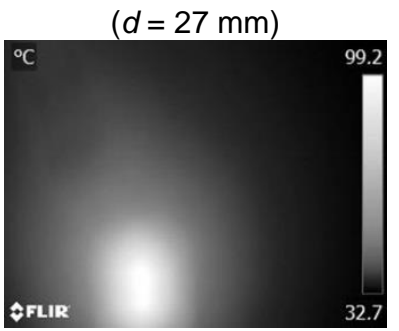

b) $\Delta \vartheta_{m}=0.1^{\circ} \mathrm{C}$ for $\mathrm{EOL}=1\left(\alpha=13.5^{\circ}\right)$

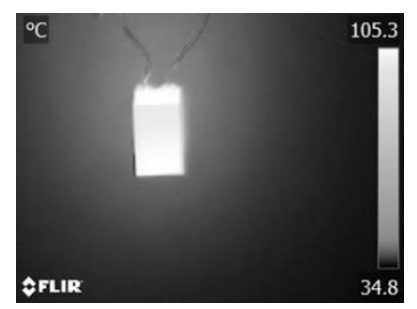

e) $\Delta \vartheta_{m}=0.1^{\circ} \mathrm{C}$ for $\mathrm{EOL}=1$ $(d=33 \mathrm{~mm})$

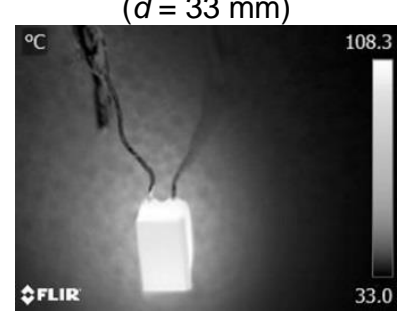

c) $\Delta \vartheta_{m}=5.04^{\circ} \mathrm{C}$ for $\mathrm{EOL}=0.061$

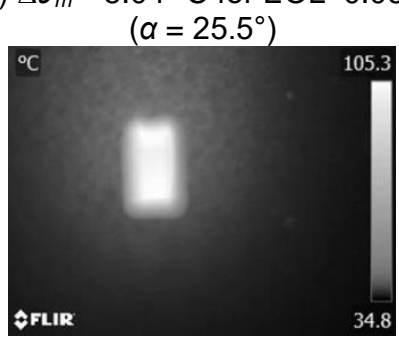

f) $\Delta \vartheta_{m}=4.66^{\circ} \mathrm{C}$ for $\mathrm{EOL}=0.057$

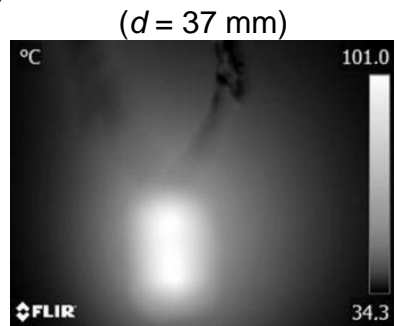

Fig. 9. Examples of thermograms with the corresponding normalized EOL value. Change in sharpness was obtained by changing $\alpha . \vartheta_{s}=101.6$

\section{Conclusions}

The knowledge of the temperature of semiconductor components is an important information in the process of designing and exploitation of electronic devices. The most important is the temperature of a semiconductor junction. However, obtaining this information is normally impossible. Therefore, as an alternative, the temperature of the semiconductor element's housing is measured, and then the temperature of the semiconductor junction is estimated on this basis. But also the measurement of the housing temperature, especially a small one, is not easy. A convenient and apparently easy way to measure the temperature of a housing is thermovision observation. However, the thermovision temperature measurement is a complex task. The result of such a measurement depends on various factors, including: emissivity factor, viewing angle, reflected radiation, obtaining a thermogram with the diagnosed element. These factors 
include the unsharpness of thermogram caused by incorrect angular position $\alpha$ of the focusing adjustment ring (inadequate to the distance $d$ between the lens and the element observed ) and small depth of field of thermal imaging cameras.

The paper presents the results of studies on the effect of thermogram unsharpness on the temperature measurement error. The graphs of relationship $\Delta \vartheta_{m}=f\left(V^{\prime}\right)$ define the quantitative effect of unsharpness expressed by the mathematical sharpness measure EOL on the absolute temperature measurement error. The determination of this relationship was preceded by the preparation of a laboratory test bench that allows for a thermovision observations of the Pt1000 sensor. The test bench was built in such a way as to limit the environmental impact on the results of temperature measurements. The tests used the Flir E50 camera with additional macro Close-up Lens 2x 197214. During the measurements, the unsharpness of thermograms was affected by changing the distance $d$ between the lens and the observed element or by changing the angular position $\alpha$ of the focusing adjustment ring. The thermograms of the observed sensor were gathered in six series of measurements - three series for a variable distance $d$ and three series for a variable angular position $\alpha$. Then the mathematical sharpness measures singled out (on the basis of literature review) were compared with the results of a survey of thermogram sharpness assessment by 107 volunteer observers. Taking into account the conclusions from this comparison and implementation simplicity, the EOL measure was selected. The last stage of works performed was to determine the value of an error of thermovision temperature measurement as a difference between the temperature measured by thermovision and the temperature of a sensor determined on the basis of its resistance. The issue of a small depth of field is shown by the characteristics of figure $7 \mathrm{~b}$ and $8 \mathrm{~b}$. While observing the PCB with embedded elements, individual elements are at different distances from the lens. In the case of a larger element, individual fragments of the housing of this element are at different distance. It can be read from figure $7 \mathrm{~b}$ that $\mathrm{a}$ change in distance $d$ by $5 \mathrm{~mm}$ will cause a change in the result of temperature measurement by about $7^{\circ} \mathrm{C}$

\section{REFERENCES}

[1] Venegas P., Usamentiaga R., Perán J., Sáez de Ocáriz I., Advances in RGB Projection Technique for Thermographic NDT: Channels Selection Criteria and Visualization Improvement, International Journal of Thermophysics. - Vol. 39, no 4, 2018.

[2] Soldan S., On extended depth of field to improve the quality of automated thermographic measurements in unknown environments, Quant. Infrared Thermogr. J. - Vol. 9, pp. 135-150, 2012.

[3] Dziarski K., Hulewicz A., Dombek, G., Frąckowiak, R., Wiczyński G. Unsharpness of Thermograms in Thermography Diagnostics of Electronic Elements. Electronics. - Vol. 9, pp. 897-912 2020.

[4] Ray SF., Applied photographic optics: lenses and optical systems for photography,film, video, electronic and digital imaging, Oxford: Focal Press, 2002.

[5] DeValois RL, DeValois KK. Spatial vision. Oxford: Oxford University Press; 1990.

[6] Pt1000 resistance temperature sensor datasheet [access: 11.05.2020, 4.53 A.M.] https://www.tme.eu/Document/67cf717905f835bc5efcdcd56ca3a8e2/Pt1000-550 EN.pdf

[7] Flir E50 datasheet [access: 11.05.2020, 4.53 A.M.] http://www.thermokameras.com/Verkauf/Flir\%20eSeri/Datenblatt\%20FLIR\%20E50\%20engl.pdf

[8] Close-up Lens 2x 197214 datasheet [access: 11.05.2020,.4.53 A.M.] http://www.ideadigitalcontent.com/files/12228/T197214-en-US A4.pdf

[9] Das K., Majumdar G., Bhowmik M. K. "Qualitative measures of breast thermograms towards abnormality prediction", 8th International Conference on Computing, Communication and Networking Technologies (ICCCNT), pp. 1-6, Delhi (India), 2017.

[10] Faundez-Zanuy M., Mekyska J., Espinosa-Duró. V., On the focusing of thermal images, Pattern Recognit. Lett. Vol. 32, pp. 1548-1557, 2011.

[11] Moorthy AK., Bovik AC., Blind image quality assessment: From natural scene statistics to perceptual quality. IEEE Trans. Image Process. - Vol. 20, pp. 3350-3364, 2011.

[12] Hassen R., Wang Z., Salama M., Image sharpness assessment based on local phase coherence, IEEE Trans. Image Process. - Vol. 22, pp. 2798-2810, 2013.

[13] Ferzli R. Karam, L., A no-reference objective image sharpness metric based on the notion of just noticeable blur (JNB), IEEE Trans. Image Process. - Vol. 18, pp. 717-728, 2009.

[14] Narvekar N., Karam L., A no-reference image blur metric based on the cumulative probability of blur detection (CPBD), IEEE Trans. - Vol 99, pp. 2678-2683, 2011.

[15] Vu C., Phan T., Chandler D., S3: A spectral and spatial measure of local perceived sharpness in natural images. IEEE Trans. Image Process. - Vol. 21, pp. 934-945, 2012.

[16] Mittal A., Moorthy A., Bovik A., No-reference image quality assessment in the spatial domain. IEEE Trans. Image Process. - Vol. 21, pp. 4695-4708, 2012.

[17] Usamentiaga R., Venegas P., Guerediaga J., Vega L., López I., A quantitative comparison of stimulation and post-processing thermographic inspection methods applied to aeronautical carbon fibre reinforced polymer, Quant. Infrared Thermogr. J. - Vol. 10, pp. 55-73, 2013.

[18] Wei H., Zhongliang J., Evaluation of focus measures in multi-focus image Fusion, Pattern Recognit. Lett. - Vol. 28, pp. 493-500, 2007. 
[19] Wang X., Wang Y. "A new focus measure for fusion of multi-focus noisy images", Proceedings of the IEEE International Conference on Computer, Mechatronics, Control and Electronic Engineering (CMCE), pp. 251254, Changchun, (China), 2010.

[20] Litwa, M. Influence of Angle of view on temperature measurements using thermovision camera. IEEE Sens. J. Vol. 10, pp. 1552-1554, 2010. 